Julian White

\title{
Factor replacement for Australian snakebite coagulopathy: a re-evaluation?
}

Received: 27 May 2009

Accepted: 3 June 2009

Published online: 23 June 2009

(C) Springer-Verlag 2009

This editorial refers to the article available at: doi:10.1007/s00134-009-1556-7.

J. White (๘)

Toxinology Department, Women's and Children's Hospital, North Adelaide, SA 5006, Australia

e-mail: julian.white@adelaide.edu.au
The paper by Brown et al. [1], on behalf of other Australian Snakebite Project (ASP) investigators and contributors, indicates a potential change in best practice management of Australian snake bite coagulopathy (venom-induced consumptive coagulopathy [VICC]). For many years, the recommendations on treatment for this concerning and potentially lethal effect of envenoming have mostly been to give adequate antivenom at the earliest indication, but to avoid using blood products (fresh frozen plasma [FFP], cryoprecipitate) to replace depleted factors, except in cases of major and catastrophic bleeding [2-5]. This advice was based on both clinical experience and a theoretical understanding of the envenoming processes involved. The exception to this was intensive care unit (ICU) teaching, where FFP was advocated as routine therapy [1]. So what has changed? Firstly, techniques for measuring venom and antivenom levels in blood samples collected serially from envenomed patients have delivered new levels of understanding of the envenoming process [6-9]. This has been linked to models of coagulation, partially developed using data from snake bite cases and "validated" using similar clinical data, which appear to indicate that the envenoming process in coagulopathy, at least for Australian snakes, involves a very short period of active venom effect [8]. This realisation, which was unexpected and remains controversial, opens the door for more aggressive use of FFP in reversing VICC, as there is no reason to delay while waiting for antivenom to reverse coagulopathy. In the past it was assumed, on the basis of clinical and laboratory observation, that reversal by antivenom was not instantaneous and that further doses might be needed. In that setting, giving FFP was considered likely to be counterproductive, adding "fuel to the fire", and the basis for generally recommending against use of FFP. A further clinical observation was the infrequency of spontaneous major bleeding associated with the coagulopathy. The current paper by Isbister et al. examines prospectively collected ASP clinical data, measured against retrospective case data, to see if existing data supports the early and more common use of FFP in treating VICC. As the authors note, their study does support FFP as likely to be effective at more rapidly returning coagulation towards a safe range. It does not address the issue of risks associated with FFP, versus the benefits. More importantly, it is only directly relevant to Australian snake bite and should not be extrapolated to snake bite coagulopathy treatment outside Australia. FFP to treat coagulopathy caused by nonAustralian venomous snakes has only rarely been tested clinically, being found ineffective for Malayan pit viper envenoming [10]. Heparin has also been found ineffective for bites by carpet vipers [11]. Australian snake venoms contain unique procoagulant toxins with no exact matches in toxin class in snake species outside Australia $[5,9,12]$. Attempts to apply the findings of this study to the clinical care of patients envenomed by nonAustralian snakes would be inappropriate, possibly dangerous, and inconsistent with best practice treatment. It may be that similar detailed studies of selected nonAustralian snakes causing coagulopathy, albeit by slightly 
or radically different toxin mechanisms, may yield evidence to change treatment practice, but the current study should not be used to drive such clinical change. Further, the current study is only one step in the process of validating changed treatment of VICC in Australia. The next logical step, a randomised control trial of FFP versus no FFP in treating VICC, is already underway. It may show that FFP speeds recovery by a few hours, but confirmation of that must await completion of the study and its publication in the peer-reviewed literature. The present study is not grounds for changing current advice on best treatment practice and doctors should refrain from "jumping the gun" and instituting changed practice ad hoc, by starting to routinely use FFP in VICC. Such a change, before all the evidence is available, would not serve the interests of patient care. It may be that when the randomised control trial of FFP for VICC is reported, it will show that FFP can hasten recovery of coagulation parameters. This is quite different from demonstrating either the safety of FFP in routine use, or improved outcomes for patients. Use of FFP has a cost to the health system. Many years of experience with VICC indicates that spontaneous bleeding, sufficient to cause concern, is uncommon to rare and major adverse bleeding events are clearly rare. Will speeding recovery of coagulation parameters actually be associated with improved outcomes for patients? The available data does not support such a contention. There are known risks using any blood product. Will these known risks outweigh any potential, possibly marginal, benefit? However, if FFP is shown to be "safe" to use early in coagulopathy, once antivenom has been given, this will be of potential benefit to a small but important subgroup of patients who do develop major bleeding problems. The ability to administer FFP soon after giving adequate antivenom in such cases may, in some situations, significantly improve outcomes. Another important issue is the value and use of antivenom. The study authors, in other published work, have indicated that antivenom may be of uncertain value in reversing VICC in most clinical settings [7-9]. This should not persuade doctors to abandon antivenom in favor of FFP, as antivenom is a multi-facetted antidote against the rich cocktail of toxins that comprise snake venom. A number of these toxins have other, potentially lethal effects, such as myolysis and neurotoxicity, that certainly require reversal if optimal patient outcomes are to be realised. Intensive care medicine alone cannot deliver optimal outcomes for such problems, without using antivenom.

\section{References}

1. Brown SGA, Caruso N, Borland ML, McCoubrie DL, Celenza A, Isbister GK (2009) Clotting factor replacement and recovery from snake venom induced consumption coagulopathy. Intensive Care Med. doi:

10.1007/s00134-009-1556-7

2. Herrmann RP, Davey MG, Skidmore PH (1972) The coagulation defect after envenomation by the bite of the Dugite (Demansia nuchalis affinis), a Western Australian brown snake. Med J Aust 2:183-186

3. White J (1987) Elapid snakes: management of bites. In: Covacevich J, Davie P, Pearn J (eds) Toxic plants and animals: a guide for Australia. Queensland Museum, Brisbane, pp 431-457

4. White J (1995) Clinical toxicology of snakebite in Australia and New Guinea. In: Meier J, White J (eds) Handbook of clinical toxicology of animal venoms and poisons. CRC Press, Boca Raton, pp 595-617
5. White J (2005) Snake venoms and coagulopathy. Toxicon 45:951-967

6. O'Leary MA, Isbister GK, Schneider JJ, Brown SG, Currie BJ (2006) Enzyme immunoassays in brown snake (Pseudonaja spp.) envenoming: detecting venom, antivenom and venom-antivenom complexes. Toxicon 48:4-11

7. Isbister GK, O'Leary MA, Schneider JJ, Brown SG, Currie BJ, ASP Investigators (2007) Efficacy of antivenom against the procoagulant effect of Australian brown snake (Pseudonaja sp.) venom: in vivo and in vitro studies. Toxicon 49:57-67

8. Tanos PP, Isbister GK, Lalloo DG, Kirkpatrick CM, Duffull SB (2008) A model for venom-induced consumptive coagulopathy in snake bite. Toxicon 52:769-780

9. Isbister GK (2009) Procoagulant snake toxins: laboratory studies, diagnosis, and understanding snakebite coagulopathy. Semin Thromb Hemost 35:93-103
10. Reid HA, Chan KE, Thean PC (1963) Prolonged coagulation defect (defibrination syndrome) in Malayan viper bite. Lancet 1:621-626

11. Warrell DA, Pope HM, Prentice CRM (1976) Disseminated intravascular coagulation caused by the carpet viper (Echis carinatus); a trial of heparin. Brit J Haematol 33:335-342

12. Kini RM (2005) Structure-function relationships and mechanism of anticoagulant phospholipase A2 enzymes from snake venoms. Toxicon 48:1147-1161 